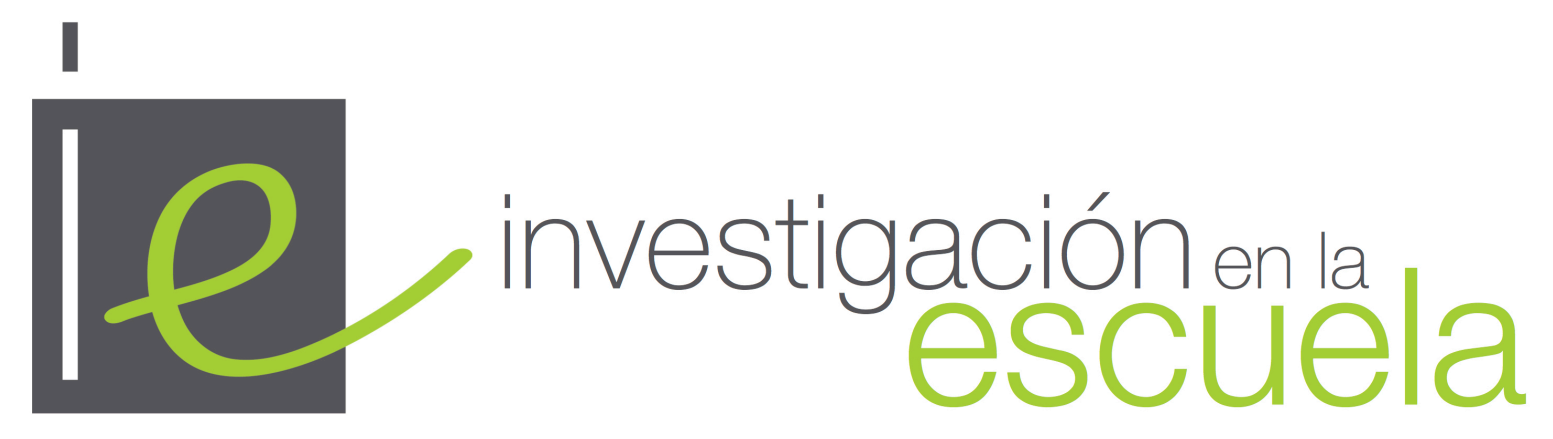

Revista internacional de investigación e innovación educativa

Número 92

07 de septiembre de 2017

ISSN 2443-9991

\title{
Una representación gráfica de la práctica de resolución de problemas en Cálculo diferencial
}

\author{
Nehemias Moreno Martinez. \\ Universidad Autónoma de San Luis Potosí \\ México
}

Citación: Moreno Martínez, N. (2017). Una representación gráfica de la práctica de resolución de problemas en cálculo diferencial. Investigación en la Escuela, 92, 60-75. Recuperado de:

http:/ / www.investigacionenlaescuela.es/articulos/R92/R92-5

Resumen: Se presenta un estudio en el que se analiza la práctica de resolución de un conjunto de problemas de Cálculo diferencial por parte de un docente de ingeniería. Los diferentes objetos matemáticos involucrados en la resolución del problema, obtenidos a partir de la producción oral y escrita del docente, son representados gráficamente mediante mapas conceptuales. Dicha representación permite, por un lado, visualizar a los diversos conceptos y sus interrelaciones puestos en juego en la resolución del problema, y, por otro lado, tener un acercamiento a los significados asociados por el sujeto.

Palabras clave: "Mapa Conceptual”; "representación gráfica”, "resolución de problemas”, “objetos matemáticos".

\section{A graphic representation of the practice of problem solving in Differential Calculus}

Abstract: You will find here a study in which the practice of solving a set of problems in Differential Calculus by an Engineering Professor is analyzed. The different mathematical objects involved in the problem solving, obtained from the oral and written production of the professor, are graphically represented through conceptual maps. Such representation allows on the one hand, visualizing the diverse concepts and their interrelations brought into play in the problem solving, and on the other hand, having an approach to the meanings associated by the subject. 
Key words: "Conceptual Map"; "graphical representation"; "problem solving"; "mathematical objects configuration".

\section{Une représentation graphique du practice de résolution des problèmes dans Calcul Différentiel}

Resumè: On présente une étude dans lequel on analyse le practice de résolution des problèmes de Calcul Différentiel par un professeur d'ingénierie. Les différents objets mathématiques impliqués dans la résolution du problème, et obtenus à partir de la production orale et écrite du professeur, sont représentés graphiquement par cartes mentales. Telle représentation permet, d'un côté, de visualiser les divers concepts et ses interrelations mis en jeu dans la résolution du problème, et d'un autre côté, d'avoir un rapprochement aux significations associées par le sujet.

Mostsclé: "Cartes mentales"; "représentation graphique"; "résolution des problèmes"; "objects mathématiques".

\section{Introducción}

En la literatura podemos encontrar diversas aplicaciones del mapa conceptual, por ejemplo, ha sido empleado como instrumento para representar la estructura cognoscitiva de los sujetos para medir los aprendizajes, en el diseño de estrategias de instrucción y de contenidos curriculares para favorecer una construcción adecuada de conocimientos, en el análisis de los conceptos más importantes para la comprensión de alguna disciplina, para negociar significados entre el docente y los alumnos, como ayuda para aprender a aprender, o como estrategia de instrucción (García, 1992; Gorbaneff y Cancino, 2009; Morales, 1998; Moreira y Novak, 1988), por mencionar algunas. Otros investigadores han señalado que el adiestramiento previo en mapas conceptuales permite la comprensión del texto del problema (González, 1993) y facilitan la evaluación del conocimiento de los alumnos (Costamagna, 2001). También se ha encontrado que existe una correlación entre la elaboración de mapas y las calificaciones favorables obtenidas en exámenes finales (Gallego, Crisol y Gámiz, 2013).

Por otro lado, también se han señalado ciertas dificultades ligadas al empleo del mapa conceptual para representar las prácticas de resolución de problemas. Según esta perspectiva, el mapa conceptual como instrumento instructivo podría ser más relevante en las Ciencias Sociales, la Biología o la Química, que, en las ciencias duras como las Matemáticas o la Física, pues éstas últimas requieren mayor aportación de conocimiento procedimental (López, 1991).

En contraste con la postura anterior, en el presente trabajo se propone al mapa conceptual, concretamente a los mapas conceptuales híbridos, como una herramienta que permite la representación gráfica de la práctica de resolución de los problemas de la matemática escolar. Se parte de la idea de separar la técnica de la teoría que apoya a los mapas conceptuales, como estrategia para reconstruir conceptualmente al mapa conceptual como objeto de investigación y mostrarlo visible desde otras perspectivas teóricas, en nuestro caso, desde el Enfoque Ontosemiótico (EOS a partir de ahora) (Godino, Batanero y Font, 2007).

Además de esta introducción, el trabajo incluye en la segunda sección el marco teórico y la metodología empleada en la investigación. En dicha sección se presenta en un primer momento el marco teórico que considera algunos elementos teóricos del EOS, le siguen algunas indicaciones sobre la técnica del mapa conceptual y posteriormente se describe la interpretación que se hace de dicha técnica a la luz del EOS. Posteriormente, se describe la metodología implementada en la investigación. 
En la tercera sección se presentan los resultados y la discusión. En la misma se describe e interpreta a los mapas conceptuales obtenidos a partir de la producción oral y escrita de un docente universitario cuando resuelve un conjunto de problemas de Cálculo diferencial. En dicho análisis se ha tomado en cuenta la perspectiva ontosemiótica de la red de conceptos representada mediante los mapas conceptuales híbridos. Por último, en la cuarta sección se presentan algunas conclusiones e implicaciones para la enseñanza.

\section{Marco teórico y metodología}

\section{Marco Teórico}

El enfoque teórico considera al mapa conceptual desde la perspectiva del EOS. A continuación, se describe primeramente el EOS, posteriormente se discute la técnica del mapa conceptual, y, por último, se incluye una interpretación de la técnica del mapa conceptual desde la perspectiva del EOS.

El Enfoque Ontosemiótico. Desde la perspectiva del EOS (Godino, Batanero y Font, 2007), la resolución de un problema matemático implica la realización de una práctica en la que participa un conjunto de objetos matemáticos primarios: conceptos, lenguaje, propiedades, procedimientos y argumentos. Las relaciones entre dichos objetos son modeladas en el EOS a través de la llamada configuración de objetos matemáticos primarios. En el EOS, tanto los objetos primarios como las configuraciones pueden ser interpretados desde cinco perspectivas duales: personal/institucional, ostensivo/no-ostensivo y expresión/contenido, unitario/sistémico e intensivo/extensivo.

Según el EOS, diversos procesos cognitivos también son llevados a cabo a lo largo de la práctica de resolución del problema y se encuentran asociados a estas perspectivas duales. Los procesos de materialización-idealización se encuentran asociados a las perspectivas ostensivo/noostensivo, puesto que en general los objetos matemáticos no son perceptibles (no ostensivos) y al mismo tiempo pueden ser observados a través de diversos objetos ostensivos asociados tales como notaciones, símbolos, gráficas, etc. A través del proceso de idealización, un objeto ostensivo del mundo material de las experiencias humanas es convertido o pensado como un objeto no ostensivo (Font y Contreras, 2008). Y a la inversa, a través del proceso de materialización, un objeto matemático pensado por un sujeto puede ser representado de manera ostensiva sobre el papel y ser observado públicamente. El vínculo entre ambos objetos (entre el objeto ostensivo y el no ostensivo) es establecido a través de una función semiótica.

También los procesos de particularización-generalización se encuentran asociados con las perspectivas extensiva/intensiva. El proceso de generalización (o abstracción) es aquel que permite al sujeto ir de un objeto extensivo (por ejemplo, $y=2 x+3$ ) a un objeto intensivo (es decir, $y=m x+b$ ), y el proceso inverso es realizado mediante la particularización (Font y Contreras, 2008). Otros procesos cognitivos también son llevados a cabo: el proceso de argumentación (que permite justificar el procedimiento empleado en la resolución de un problema), visualización (Godino, Gonzato, Cajaraville y Fernández, 2012), representación, entre otros.

Por otra parte, el significado es entendido en el EOS como "función semiótica" o como "sistema de prácticas". Mediante el primero, se considera la perspectiva expresión/contenido, el significado está dado a través de la correspondencia (relaciones de dependencia) entre un antecedente (expresión) y un consecuente (significado o contenido) establecidos por un sujeto (persona o institución) de acuerdo a ciertos criterios (convenios, reglas matemáticas). Mediante el segundo, el significado es entendido como el sistema de prácticas en las que dicho objeto es 
determinante para su realización. En este último, el sistema de prácticas se puede parcelar en diferentes clases de prácticas más específicas que son utilizadas en un determinado contexto y con un determinado tipo de notación produciendo un determinado "sentido" (perspectiva unitaria) del objeto matemático.

Los Mapas Conceptuales. El mapa conceptual es una red de conceptos ordenados jerárquicamente, en el que la interconexión de los conceptos, mediante los "nexos" y las "frases de enlace", produce una red de estructuras proposicionales donde el significado no sólo se encuentra en la relación entre concepto y concepto, sino que se extiende a las relaciones que a su vez estos conceptos tienen con otros; el orden de estas relaciones está orientado por un dominio de conocimiento a partir del cual es posible señalar las relaciones verdaderas conforme al conocimiento de referencia. El mapa conceptual puede ser elaborado a partir de un texto mediante una transformación de los registros (Aguilar, 2006).

Por otro lado, el desarrollo del mapa conceptual y su implementación en otros campos de conocimiento ha dado lugar a la fusión del mapa conceptual con otro tipo de representaciones, tal es el caso de los mapas conceptuales híbridos que resultan de la fusión de la red jerárquica de conceptos -característica del mapa conceptual-, con la representación gráfica de procesos -característica de un diagrama de flujo-.

En este trabajo se realiza la propuesta de emplear al mapa conceptual híbrido para describir de manera gráfica la práctica de resolución de problemas de las matemáticas en etapa escolar. En el mapa conceptual híbrido, la componente del mapa conceptual permite representar objetos matemáticos tales como conceptos, propiedades y argumentos, mientras que la componente del diagrama de flujo permite la representación del objeto procedimiento, el cual involucra a procesos matemáticos tales como el de tratamiento algebraico, numérico, entre otros.

Una mirada al mapa conceptual desde el Enfoque Ontosemiótico. Desde la perspectiva del EOS, el mapa conceptual híbrido es una representación ostensiva de la práctica de resolución de un problema de las matemáticas escolares. El mapa conceptual híbrido considera: (1) lenguaje, que permite representar de manera ostensiva objetos no ostensivos (por ejemplo, conceptos a través de nombres, propiedades mediante expresiones matemáticas, etc.); (2) conceptos, que se organizan jerárquicamente en el mapa conceptual híbrido; (3) propiedades, que se expresan mediante rutas de lectura que conforman enunciados sobre conceptos (por ejemplo, propiedades algebraicas o geométricas); (4) procedimiento, representado a través de la componente procedimental del diagrama de flujo en el mapa conceptual; y (5) argumentos, obtenidos de las diferentes rutas de lectura que conforman enunciados que validan o explican las proposiciones y procedimientos deductivos o de otro tipo.

Los mapas conceptuales representan la organización jerárquica de los conceptos, la cual va de los conceptos de mayor a menor generalidad. El paso de una jerarquía a otra conlleva la realización de ciertos procesos tales como el de idealización, argumentación, tratamiento, entre otros. El mapa conceptual también puede ser visto desde la perspectiva personal/institucional, según se trate de un mapa conceptual obtenido a partir de las producciones de un experto (perspectiva institucional) o de un estudiante inexperto (perspectiva cognitiva) respectivamente.

En relación con el significado, se considera la perspectiva unitaria y sistémica. Desde la perspectiva unitaria, las representaciones que se muestran en el mapa conceptual dan cuenta del establecimiento de funciones semióticas establecidas por el sujeto que resuelve el problema. Por ejemplo, los conceptos matemáticos pueden ser representados en el mapa conceptual mediante un nombre (perspectiva ostensiva) o mediante una expresión matemática, por ejemplo, el concepto de 
"pendiente" de una recta puede estar expresado mediante " $m=\tan (\alpha)$ ". Las funciones semióticas también son establecidas a través de la articulación entre conceptos, es decir, a través de una proposición que enuncia una propiedad o un argumento justificativo.

El significado de un objeto matemático también es entendido como un sistema de prácticas en el que dicho objeto es determinante (perspectiva sistémica). Esto es, tomando en cuenta que el mapa conceptual híbrido representa a la práctica de resolución de un problema matemático, un concepto en un mapa conceptual puede ser interpretado de una manera distinta en otro mapa (del sistema de mapas). Por ejemplo, en un mapa conceptual la derivada de una función puede ser concebida como la pendiente de la recta tangente a un punto de la gráfica de dicha función, sin embargo, en otro mapa conceptual que represente a una práctica más general, la derivada podría ser interpretada como la razón de cambio o tasa de variación de alguna cantidad a un tiempo dado.

\section{Metodología}

La investigación toma en cuenta un estudio cualitativo, puesto que se trata de un estudio de tipo exploratorio en el que se considera la observación del tipo de solución a las tareas propuestas. La estrategia de indagación empleada en este trabajo fue un estudio de caso (Merrian, 1998) debido a que se tenía el interés de describir e interpretar la práctica de resolución de un conjunto de problemas matemáticos escolares. El estudio de caso es descriptivo, pues se presentan con detalle las producciones (oral y escrita) realizadas por el sujeto a lo largo del proceso de la resolución de cada una de las tareas planteadas. También es interpretativo en el sentido de que los datos recabados son empleados para desarrollar categorías conceptuales a manera de ilustrar la pertinencia del empleo de la perspectiva teórica del EOS para representar gráficamente la práctica de resolución de problemas.

En la resolución de las tareas propuestas, el docente realiza una práctica matemática que consiste en la lectura del texto donde se enuncia el problema y en la producción de un discurso oral y escrito con la respuesta a la cuestión planteada, la cual será el resultado de una serie de acciones sujetas a reglas matemáticas.

Participante. El participante fue un docente universitario con formación académica en ingeniería mecánica y con estudios de posgrado en ciencias aplicadas. El docente informó que contaba con cinco años de experiencia impartiendo la materia de Cálculo en la Universidad Autónoma del Estado de Morelos, México.

Diseño y recogida de datos. El investigador diseñó dos problemas que fueron nombrados como "el problema del globo" y "el problema de la caja de madera", los cuales son abordados típicamente en los cursos de Cálculo diferencial que se imparten en la universidad a la que se encuentra adscrito el docente investigado. Los problemas fueron los siguientes:

(1) Un globo sonda se expande conforme se eleva, debido a la disminución de la presión atmosférica. Suponga que el radio " $r$ " aumenta a razón de 0.03 pulgadas por segundo y que $\mathrm{r}=48$ pulgadas en el instante $\mathrm{t}=0$. Determine una ecuación que modele el volumen " $\mathrm{V}$ " del globo en el instante " $\mathrm{t}$ " y determine el volumen cuando $t=300$ segundos.

(2) Se ha de construir una caja de madera de base cuadrada y de $108 \mathrm{dm}^{3}$ de capacidad. La parte de arriba debe ser abierta. ¿Qué dimensiones debe tener la caja para que la cantidad de material empleada en su construcción sea mínima? Es decir, ¿qué dimensiones exigirán menor costo?

El procedimiento para la obtención de los datos se llevó a cabo en una sola sesión entre el investigador y el docente investigado, en dicha sesión se propuso al docente la resolución de los problemas, y se le dio la oportunidad de reflexionar sobre ellos y resolverlos antes de que los 
resolviese frente al investigador mediante el empleo de la pluma electrónica. La pluma electrónica "Smartpen" es una herramienta electrónica que permite el registro sincronizado de audio y trazo de escritura, el cual es almacenado en un archivo electrónico para reproducirse en la computadora. Dicha herramienta permite tener en cuenta el discurso del docente al mismo tiempo que presenta la solución del problema sobre el papel.

La organización de los datos y la elaboración de los mapas conceptuales correspondientes fueron llevadas a cabo por el investigador y fue realizada tomando en cuenta la producción escrita y oral del docente analizado. Se elaboró una tabla en donde se clasificó y se enumeró (según el orden de aparición en la resolución del problema) a los elementos de la producción oral y escrita según si se correspondían con los objetos matemáticos señalados por el EOS en: conceptos, propiedades, argumentos y procedimientos. En cada uno de éstos, se hizo la anotación correspondiente del lenguaje empleado para expresar dichos objetos a través de nombres, literales, expresiones matemáticas, operaciones y proposiciones enunciadas por el docente.

A partir de la tabla de objetos matemáticos, el mapa conceptual iniciaba con el concepto de situación-problema como pregunta de enfoque, puesto que después de dicho objeto le siguen conceptos significativos para el docente que dan cuenta de la interpretación que da al texto donde se plantea el problema en cuestión. Cuando el sujeto se refiere a alguna propiedad matemática ésta es enunciada a través de una proposición representada mediante una ruta de lectura en el mapa conceptual en la que no se justifica el procedimiento de solución, pero si se establece una relación matemática entre conceptos. El procedimiento realizado por el sujeto es incorporado en el mapa conceptual a través de la componente del diagrama de flujo, el cual muestra la aplicación de una serie de propiedades matemáticas (propiedades algebraicas, sustituciones, despejes, leyes de signos, etc.) que guían al sujeto hacia la solución del problema. Por otra parte, la argumentación oral proporciona la componente justificativa del procedimiento de solución del problema, el cual es representado a través de la trama de rutas de lectura del mapa.

\section{Resultados y discusión}

\section{El problema del globo}

En la figura 1 se muestra la producción del docente, que consiste en el tratamiento algebraico y del discurso oral (presentado mediante el texto en recuadros), ésta última obtenida de la transcripción del audio del archivo generado con la pluma electrónica. Cabe señalar que el docente no se apoyó en otro tipo de representaciones como en esquemas o en alguna representación pictórica. 


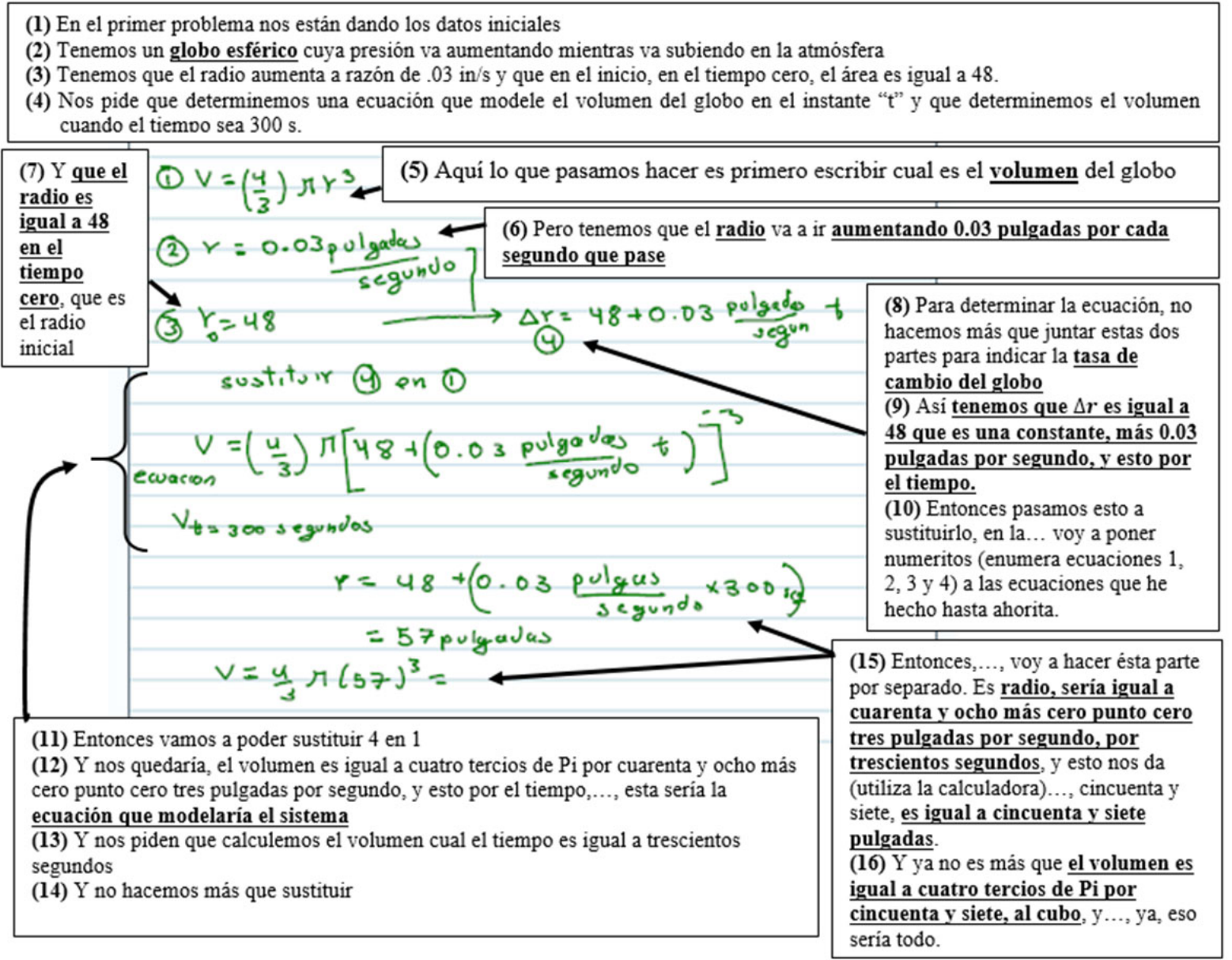

Figura 1. Producción del docente en la resolución del problema del globo

En la figura 2 se muestra el mapa conceptual híbrido correspondiente. Con el fin de ilustrar la correspondencia entre la producción del docente y el mapa conceptual, en la figura 1 se han subrayado los conceptos que fueron considerados en la elaboración del mapa conceptual de la figura 2.

En comparación con los libros de texto, que presentan el tratamiento algebraico separado del discurso justificativo y dejan al lector la lectura y la visualización de la información textual sobre el tratamiento algebraico, la perspectiva ontosemiótica de los mapas conceptuales híbridos provee de una herramienta que permite representar de manera conjunta tanto el tratamiento algebraico como la información que proporciona la transcripción del discurso oral justificativo, ver la figura 2.

En el mapa conceptual de la figura 2 se pueden observar diversos conceptos (algunos han sido señalados mediante números $1,2, \ldots, 6$ y otros de letras A, B, B1, C, D y E) articulados y organizados de manera jerárquica. Dicha jerarquía va de los conceptos que resultaron significativos para el docente a partir de la lectura del problema, como el concepto de globo (concepto 2), volumen y radio (conceptos 3 y A), llegando hasta conceptos más complejos como el de ecuación del sistema (concepto 5) o el de tasa de cambio (concepto C), los cuales son incorporados por el experto con el propósito de resolver el problema. Obsérvese la eficacia de los mapas conceptuales para revelar la práctica del docente. 


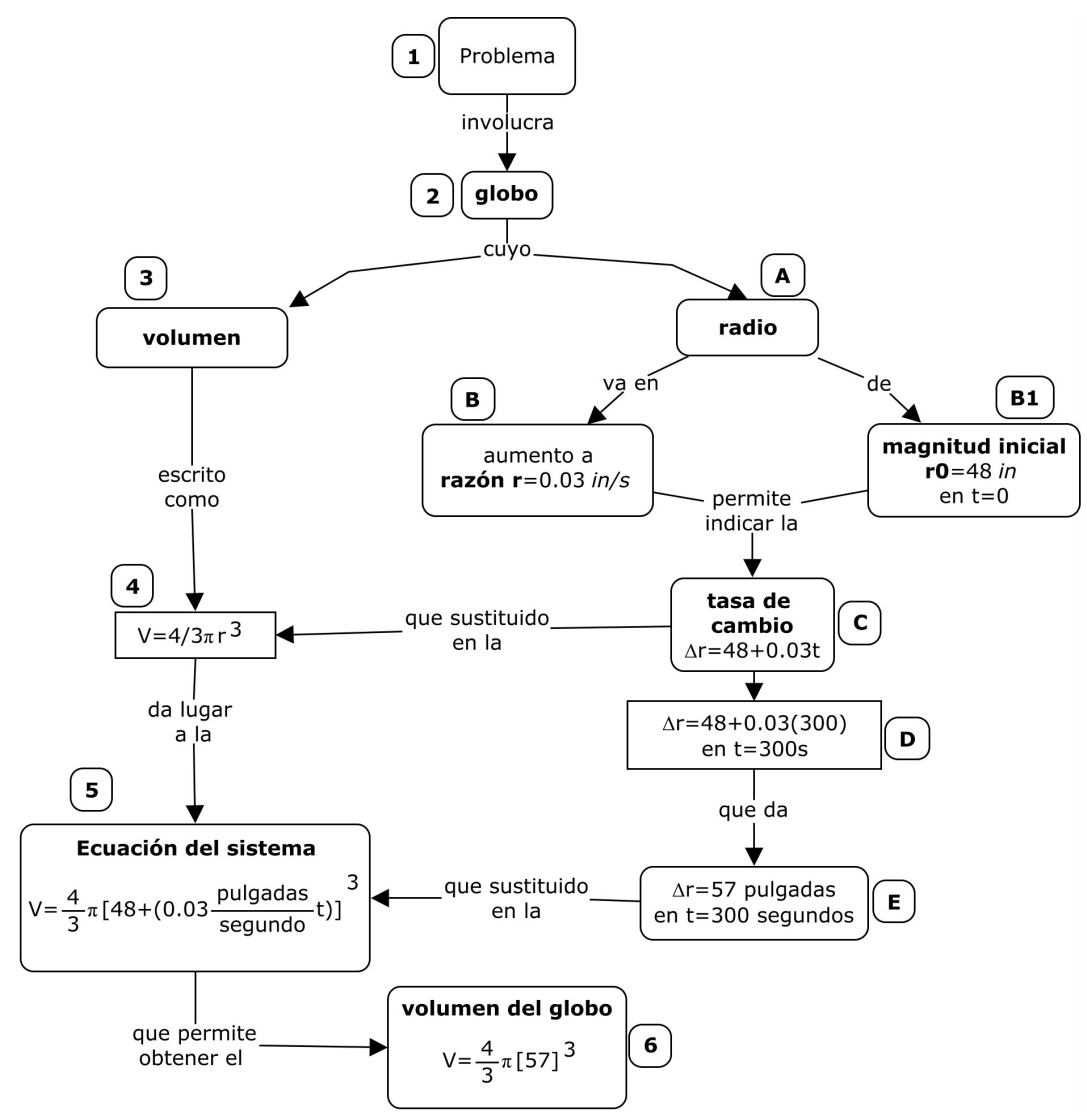

Figura 2. Mapa conceptual híbrido correspondiente a la solución del problema del globo (elaborado mediante el software Cmap'Tools)

En la figura 2 se observan diversos conceptos que son representados ya sea mediante nombres (por ejemplo: globo, radio, volumen, razón, tasa de cambio, etc.) o bien mediante expresiones matemáticas tales como el de volumen " $V=4 / 3 \pi r$ ", tasa de cambio $\Delta r=48+0.03 t$ o la ecuación del sistema " $V(t)=4 / 3 \pi[48+0.03 t]$ ". Todas estas representaciones dejan ver el establecimiento de una trama de funciones semióticas entre las expresiones, nombres o expresiones algebraicas, y contenidos o conceptos a los que se hace referencia.

Cabe señalar también la incorporación de otros conceptos tales como "sistema" (ecuación del sistema, concepto 5) o el de tasa de cambio (concepto C), que hacen referencia a los conocimientos previos del docente. También se observa el manejo implícito de conceptos y operaciones tales como el concepto de función (conceptos 4, C y 6) o la operación de composición de funciones (conceptos C-4-5).

También es posible observar el empleo de algunas propiedades, por ejemplo, la propiedad señalada por la ruta de lectura que va del concepto A al B (ruta A-B) que dice que "el radio va en aumento a razón $r=0.03 \mathrm{in} / \mathrm{s}$ ", también las propiedades que hacen posible la composición de funciones reales mediante la ruta C-4 "la tasa de cambio $\Delta r=48+0.03 t$ sustituida en la función $V=4 / 3 \pi r^{3}$ " o bien la propiedad señalada en la ruta A-B1 que se refiere a la condición inicial "el radio de magnitud inicial $r_{0}=48$ in en $t=0$ '.

Asimismo, la figura 2 también deja ver la realización de un procedimiento que involucra en un primer momento la escritura del volumen del globo esférico en función del radio " $V=4 / 3 \pi r^{3}$ " (ruta 2-4), en un segundo momento "juntar" los conceptos B y B1 para deducir la ecuación del radio del globo (el docente llama a esta función tasa de cambio) en función del tiempo " $\Delta r=48+0.03 t$ " 
(conceptos A-B-B1-c). Después, sin señalarlo explícitamente, realiza la composición de las funciones $V$ y $r$ (ruta C-4-5), que le permite determinar el volumen del globo en función del tiempo, y el volumen al tiempo $\mathrm{t}=300 \mathrm{~s}$ (concepto 6 ).

Pese a que el docente llega al resultado correcto, cabe señalar que nombra a $\Delta r$ tasa de cambio y además lo interpreta como " $r$ " puesto que realiza la composición de funciones (ver $8,9,10$ y 11 en la figura 1 y conceptos C, 4 y 5 en la figura 2). Desde la perspectiva educativa, si se aborda en el aula el problema del globo como un medio para entrenar a los estudiantes en la modelación matemática de fenómenos, la práctica realizada por el docente podría plantear una serie de dificultades para el aprendizaje de los alumnos.

Lo anterior deja ver la utilidad del mapa conceptual en cuanto a que permite la visualización de la articulación de los conceptos (por ejemplo, la articulación de los conceptos B y B1 para dar lugar a C) y los significados asociados (por ejemplo, la interpretación de $\Delta r$ como " $r$ "), los cuales podrían no ser evidentes para los alumnos a través de la producción escrita del docente. El mapa conceptual también permite la visualización de aspectos de la enseñanza de las matemáticas de las cuales muchas veces los docentes no somos conscientes, como, por ejemplo, el manejo implícito de conceptos y operaciones o el empleo de notaciones inadecuadas.

También es posible observar en la figura 2 diversos argumentos que vienen a justificar el procedimiento empleado. Estos argumentos se muestran a través de las siguientes rutas de lectura: (i) ruta 1-6, el problema involucra un globo cuyo volumen se representa mediante una función $V$ (en función de " $r$ ") la cual da lugar a la ecuación del sistema $V$ (en función de " $t$ ") que permite obtener el volumen del globo en $\mathrm{t}=300$ segundos y (ii) ruta A, B, B1, C-F, el radio va en aumento a razón $r=0.03 \mathrm{in} / \mathrm{s}$ y junto con el radio inicial $r_{0}$ permite indicar la tasa de cambio $\Delta r=48+0.03 t$ de magnitud $\Delta r=57$ pulgadas en $t=300$ segundos.

Por último, en la resolución del problema también se llevan a cabo algunos procesos cognitivos. El docente realiza el proceso de idealización al pensar el globo sonda como una esfera (esto permite ir del concepto 2 al concepto 4 en la figura 2). Por otro lado, a partir del caso particular del globo, el docente lleva a cabo un proceso de generalización que le permite deducir una expresión lineal de la forma $\mathrm{f}(\mathrm{x})=\mathrm{mx}+\mathrm{b}$, para el radio como una función del tiempo $\Delta \mathrm{r}=48+0.03 \mathrm{t}$. Es decir, por medio de la generalización, los valores del radio inicial de 48 in y la razón de cambio de 0.03 in/s son pensados por el docente como parámetros característicos de una clase general de relación funcional, las funciones lineales.

También se realiza el proceso de materialización, en el sentido de que aparecen a la vista de manera secuencial distintos objetos tales como la expresión del volumen, en un primer momento en función del radio y posteriormente en función del tiempo, o bien la misma solución del problema del globo $V=4 / 3 \pi(57)^{3}$. Se realizan otros procesos como el proceso de argumentación con el propósito de justificar el procedimiento empleado para resolver el problema, y el proceso de tratamiento que va del concepto $\mathrm{C}$ al $\mathrm{F}$ y que se corresponde con la componente del diagrama de flujo del mapa conceptual híbrido. Se observa que algunos procesos permiten ir de una jerarquía a otra en el mapa conceptual.

\section{El problema de la caja de madera}

En la figura 3 se ilustra la producción del docente en la que se observa el tratamiento algebraico realizado, la representación pictórica de la caja y el discurso oral presentado en recuadros. 


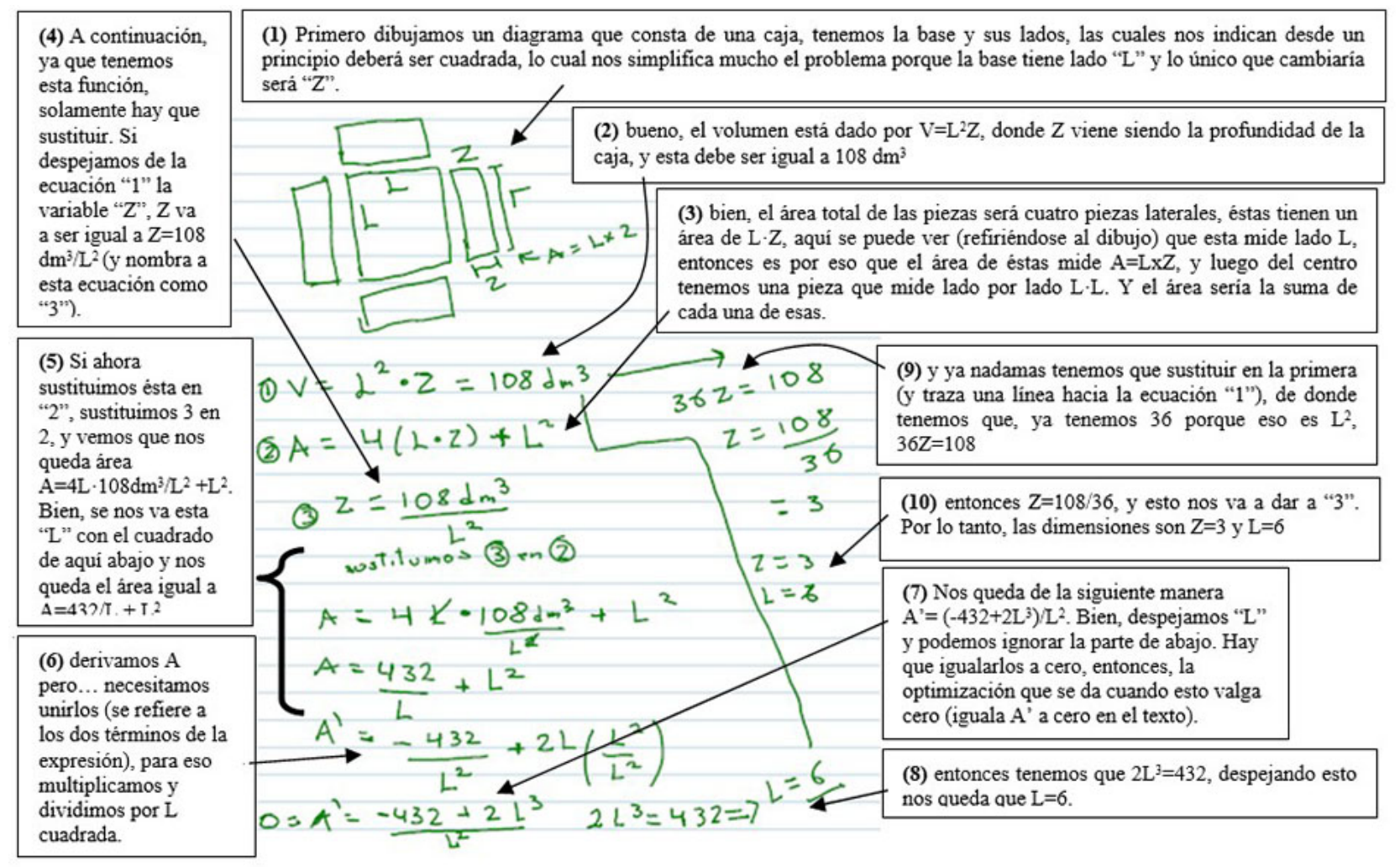

Figura 3. Producción del docente en la resolución del problema de la caja de madera

En la figura 3 se observa que el docente inicia la resolución del problema representado pictóricamente a la caja sin tapa como una figura plana y señalando algunas literales para referirse a las medidas de la base cuadrada, largo "L" y ancho "L", y a la altura "Z". En dicha representación el espesor de cada parte de la caja es considerada irrelevante.

En la figura 4 se muestra el mapa conceptual híbrido que corresponde a la producción oral y escrita del docente en la resolución del problema de la caja. El mapa conceptual muestra aquellos conceptos (conceptos 1-16, A-D, K-N y R-T, por señalar la mayoría) que resultaron significativos para el docente en la resolución del problema.

$\mathrm{Al}$ igual que el mapa de la figura 2, es posible observar una jerarquía de conceptos. En el mapa conceptual se observan conceptos (por ejemplo, conceptos 2, A, L o 16) que fueron obtenidos directamente por el docente a partir de la lectura del texto del problema. Otros conceptos (caja sin tapa, mínimo de material), que no aparecen en el mapa, también fueron tomados en cuenta por el docente y le guiaron en el procedimiento de solución de problema, tal es el caso de los conceptos de área total (concepto 3) y de centro y cuatro piezas laterales (conceptos L y K) que resultan de considerar a la caja constituida por cinco piezas y no de seis piezas (con la tapa), o bien, el hecho de prescindir del espesor de la caja e introducir el concepto de área para poder trabajar minimizando el área total de las piezas de la caja y así obtener el mínimo de material para construcción.

Por otra parte, hay conceptos como el de área, cuatro piezas laterales, centro, profundidad; propiedades como la suma de áreas (conceptos $3, \mathrm{~K}, \mathrm{~L}$ y 4 ), la optimización de una función mediante la derivada (conceptos 7-16), la multiplicación por el elemento neutro $\mathrm{L}^{2} / \mathrm{L}^{2}$ (concepto 7); y operaciones tales como la composición de funciones (conceptos D, 4-6) u operaciones algebraicas (conceptos 7-16) entre las que se encuentran las operaciones para encontrar al mínimo de una función mediante la primera derivada, las cuales aparecen en el mapa conceptual pero que no se 
encuentran señalados en el texto que describe el problema, sino más bien son incorporados por el docente al tomar en cuenta su conocimiento previo, por ejemplo, su conocimiento perceptual (por ejemplo, prescindir del espesor de las piezas de la caja) o su experiencia en la resolución de problemas (resolución de otros problemas de optimización).

En el mapa también se observa que el docente establece una trama de funciones semióticas. Se establecen relaciones semióticas entre los diversos nombres y literales (V para representar el volumen, $\mathrm{L}$ para el lado, $\mathrm{Z}$ para la profundidad, etc.) y los conceptos a los que se hacen referencia. También representa algunos conceptos mediante expresiones algebraicas tales como la del volumen $\mathrm{V}=\mathrm{L}^{2} \mathrm{Z}$ (concepto B) o la expresión de la suma de áreas $\mathrm{A}=4(\mathrm{LZ})+\mathrm{L}^{2}$ (concepto 4).

Los significados de los diversos objetos (lenguaje, conceptos, propiedades, argumentos, procedimiento) que participan en la resolución del problema de la caja están determinados por la red de relaciones semióticas señaladas en el mapa conceptual (ver la figura 4). Por ejemplo, los conceptos de base y profundidad (conceptos L y S) tienen sentido puesto que se ha hecho referencia previamente a una caja de madera que tiene cierto volumen (concepto 2 y A).

Los argumentos que pueden ser observados a través del mapa conceptual también cobran sentido cuando se les considera relacionados a otros argumentos. Por ejemplo, mediante la ruta de lectura 1-16 se señala que "el problema involucra una caja de madera que tiene un área total expresada como la suma de áreas $\mathrm{A}=4(\mathrm{LZ})+\mathrm{L}^{2}$ con las dimensiones $\mathrm{Z}=3$ y $\mathrm{L}=6$ (que minimizan el área y el material)", la cual tiene sentido si se consideran otros argumentos como el que proporciona la ruta de lectura 2, A y B que dice "caja de madera que tiene volumen dado por $\mathrm{V}=\mathrm{L}^{2} \mathrm{Z}$ " y el argumento señalado mediante la ruta de lectura $2,3, \mathrm{~K}$ y L "caja de madera que tiene área total que es la de cuatro piezas laterales y la de una base", ésta última proposición también tiene sentido si se considera la ruta K-T que señala "cuatro piezas laterales que tiene área LZ cada una”. Nótese la versatilidad de los mapas conceptuales para mostrar las diversas operaciones matemáticas realizadas por el docente. 


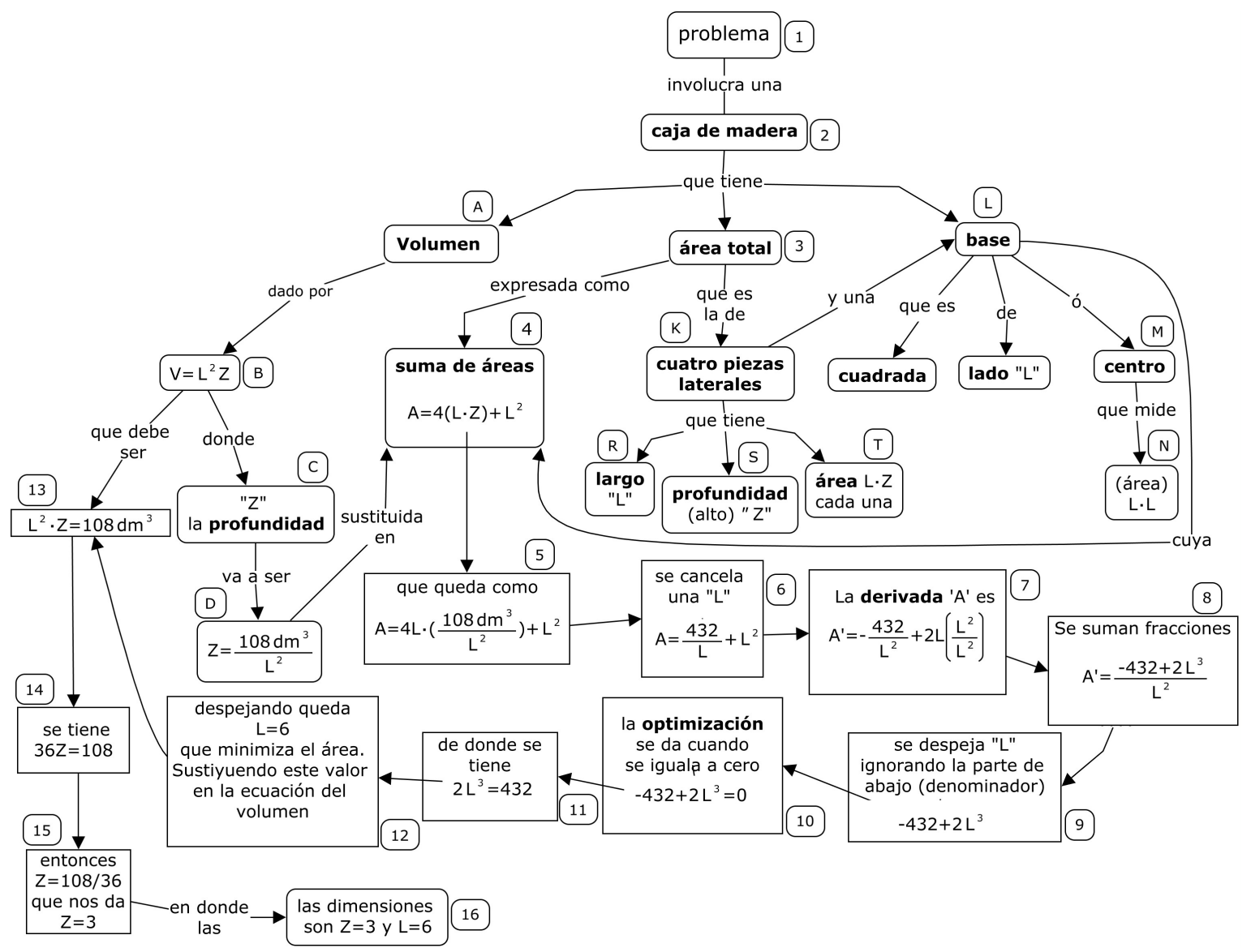

Figura 4. Mapa conceptual híbrido epistémico correspondiente a la solución del problema de la caja de madera (elaboración propia mediante CmapTools)

El mapa conceptual de la figura 4 también permite visualizar ciertos aspectos en los que el docente no consideró pertinente ahondar. Por ejemplo, en el argumento principal o tesis que señala la solución del problema, el docente no justificó por qué sólo tomó en cuenta al numerador de la función A' para poder determinar las dimensiones óptimas de la caja (objetos 9 y 10). Tampoco se apoyó en el resultado de la segunda derivada para mostrar la validez del resultado obtenido.

Por otro lado, la red de objetos que se muestra mediante formas rectangulares, objetos enumerados del 5 al 15 en el mapa conceptual de la figura 4, conforman la componente del diagrama de flujo del mapa conceptual. A diferencia de la red de conceptos de un mapa conceptual de donde es posible obtener proposiciones, en la sucesión de objetos rectangulares sólo se hace referencia al tratamiento matemático, de manera que el texto al interior de cada objeto sólo indica la operación que se está realizando en dicho módulo.

El docente nuevamente realiza el proceso de idealización cuando supone que la caja posee espesor despreciable, reduciendo el problema inicial a un problema en el plano. También realiza un proceso de visualización puesto que realiza una transformación visual de la caja al descomponerla en cinco partes o piezas planas, comunica la posición relativa de las partes de la caja haciendo uso de lenguaje deíctico para hacer referencia a la base (centro) y a la altura de la caja (profundidad). El docente lleva a cabo el proceso de argumentación, lo cual puede observarse a través de las diferentes rutas de lectura en el mapa conceptual, y de tratamiento matemático a lo largo de la secuencia de objetos rectangulares (objetos 5-15). 


\section{Conclusiones e implicaciones para la enseñanza}

Una de las conclusiones de este trabajo es que la interpretación del mapa conceptual desde la perspectiva del EOS ha permitido la descripción gráfica de la práctica de resolución de un conjunto de problemas de cálculo diferencial. En dicha interpretación, los objetos matemáticos (lenguaje, conceptos, propiedades, procedimientos y argumentos) señalados por el EOS como aquellos objetos primarios que se tienen en cuenta en la práctica de resolución de problemas matemáticos, han sido representados de manera gráfica mediante la técnica de mapas conceptuales, en particular mediante el empleo de mapas conceptuales híbridos. Los mapas conceptuales también permitieron evidenciar la realización de ciertos procesos tales como el de idealización, visualización, argumentación, tratamiento matemático, entre otros.

Por otra parte, se sabe que en la resolución de problemas en los libros de texto, el tratamiento matemático y el texto justificativo se muestran separados, dejando al lector la tarea de visualización de la información sobre la secuencia de operaciones matemáticas señaladas en algún lugar de la página del libro. En contraste, los mapas conceptuales híbridos a través del sistema de rutas de lectura permiten representar gráficamente en la misma región el tratamiento matemático y el discurso justificativo del sujeto, los mapas de las figuras 2 y 4 permiten observar esto.

La propuesta que se describe en el presente trabajo podría ser implementada en la clase para guiar a los estudiantes a lo largo del proceso de resolución de los problemas matemáticos, o bien a través de la elaboración de mapas conceptuales y la posterior discusión acerca de la estructura de estos. Por otro lado, la perspectiva personal/institucional del mapa conceptual también podría proporcionar una herramienta para analizar los conocimientos de los estudiantes en el contexto de la resolución de problemas de la matemática escolar, pues se trata de la elaboración y comparación sistemática de un mapa conceptual híbrido epistémico con el correspondiente mapa conceptual híbrido cognitivo que se desea analizar.

Cabe señalar que este trabajo presenta una forma de abordar la técnica del mapa conceptual desde la mirada del EOS. Se ha evitado la suplantación del sustento teórico, es decir, no se han confrontado los supuestos teóricos de la teoría del aprendizaje significativo de Ausubel (Coll, 1988) con respecto a los elementos teóricos del EOS. Esto último es debido a la diversidad y amplitud de las prácticas del mapa conceptual que van más allá de estas dos formas de abordar el problema del aprendizaje de la matemática escolar.

\section{Referencias}

Aguilar, M. F. T. (2006). El mapa conceptual una herramienta para aprender y enseñar. Plasticidady restauración neurológica, 5 (1), 62-72.

Coll, C. (1988). Significado y sentido en el aprendizaje escolar. Reflexiones en torno al concepto de aprendizaje significativo. Infancia y aprendizaje, 11 (41), 131-142.

Costamagna, A. M. (2001). Mapas conceptuales como expresión de procesos de interrelación para evaluar la evolución del conocimiento de alumnos universitarios. Enseñanz̧a de las Ciencias, 19 (2), 309-318.

Font, V. y Contreras, Á. (2008). The problem of the particular and its relation to the general in mathematics education. Educational Studies in Mathematics, 69 (1), 33-52.

Gallego, A. J., Crisol M. E. y Gámiz S. V. (2013). El mapa conceptual como estrategia de aprendizaje y de evaluación en la universidad. Enseñanz̧a and Teaching, 31 (2), 145-165. 
García, F. M. G. (1992). Los mapas conceptuales de JD Novak como instrumentos para la investigación en didáctica de las ciencias experimentales. Enseñanza de las Ciencias, 10 (2), 148 158.

Godino, J. D., Batanero, C. y Font, V. (2007). The onto-semiotic approach to research in mathematics education. ZDM-The International Journal on Mathematics Education, 39 (1-2), $127-$ 135.

Godino, J. D., Gonzato, M., Cajaraville, J. A., y Fernández, T. (2012). Una aproximación ontosemiótica a la visualización en educación matemática. Enseñanza de las ciencias: revista de investigación y experiencias didácticas, 30 (2), 109-130.

González, L. C. C. (1993). Mapas conceptuales y resolución de problemas. Investigación en la escuela, 19, 79-88.

Gorbaneff, Y. y Cancino, A. (2009). Mapa conceptual para el aprendizaje basado en problemas. Estudios Gerenciales, 25 (110), 111-124.

López, R.F. (1991). Organización del conocimiento y resolución de problemas en física. Madrid: Centro de Publicaciones del Ministerio de Educación y Ciencia, CIDE.

Merrian, S. R. (1998). Qualitative Research and Case Study Applications in Education. Second Edition. San Francisco: Jossey-Bass Publishers.

Morales, E. (1998). Efecto de una didáctica centrada en la resolución de problemas empleando la técnica heurística V de Gowin y mapas conceptuales en el razonamiento matemático. Revista Latinoamericana de Investigación en Matemática Educativa, 1 (2), 77-91.

Moreira, M. A. y Novak, J. D. (1988). Investigación en enseñanza de las ciencias en la Universidad de Cornell: esquemas teóricos, cuestiones centrales y abordes metodológicos. Enseñanza de las Ciencias, 6 (1), 3-18.

\section{Información sobre el autor}

Autor: Nehemías Moreno Martínez

Institución: Universidad Autónoma de San Luis Potosí

Email: nehemias_moreno@live.com 


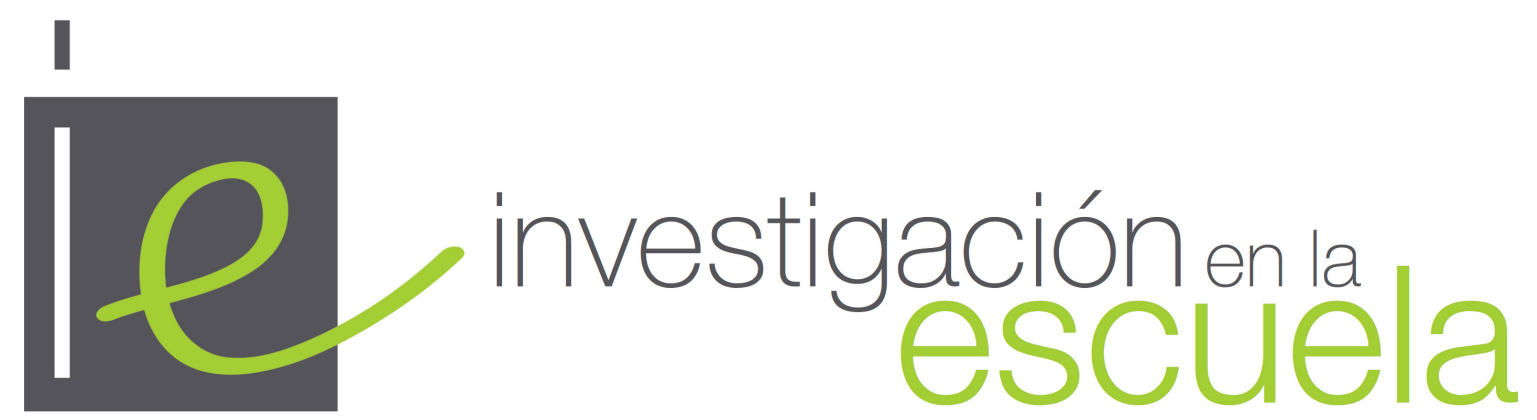

Revista académica evaluada por pares y de acceso abierto

Número 92

07 de septiembre de 2017

ISSN 2443-9991

\section{(c)}

SORERIIGHISRESERVEDLOS/as lectores/as pueden copiar, mostrar, y distribuir este artículo, siempre y cuando se de crédito y atribución al autor/es y a Investigación en la Escuela, se distribuya con propósitos no-comerciales, no se altere o transforme el trabajo original. Más detalles de la licencia de CreativeCommons se encuentran en http://creativecommons.org/licenses/by-nc-sa/3.0 Cualquier otro uso debe ser aprobado en conjunto por el autor/es, o Investigación en la Escuela.

Uิ investigacion-en-la-escuela

Contribuya con comentarios y sugerencias en la web de la revista. Por errores y sugerencias contacteasecretaria@investigacionenlaescuela.es 


\section{Investigación en la escuela}

Consejo de dirección: Ana Rivero García (Universidad de Sevilla), Nicolás de Alba Fernández(Universidad de Sevilla), Pedro Cañal de León (Universidad de Sevilla), Francisco F. García Pérez (Universidad de Sevilla), Gabriel Travé González, (Universidad de Huelva), Francisco F. Pozuelos Estrada (Universidad de Huelva)

Dirección: Ana Rivero García y Nicolás de Alba Fernández

Secretaría de edición: Elisa Navarro Medina

\section{Consejo editorial}

José Félix Angulo Rasco. Universidad de Cádiz Rosa Ma Ávila Ruiz. Universidad de Sevilla Pilar AzcárateGoded. Universidad de Cádiz Juan Bautista Martínez Rodríguez. Universidad de Granada

Nieves Blanco García. Universidad de Málaga Fernando Barragán Medero. Universidad de La Laguna José Carrillo Yáñez. Universidad de Huelva José Contreras Domingo. Universidad de Barcelona. Luis C. Contreras González. Universidad de Huelva Ana $\mathbf{M}^{\mathbf{a}}$ Criado García-Legaz. Universidad de Sevilla Rosario Cubero Pérez. Universidad de Sevilla José $\mathbf{M}^{\mathbf{a}}$ Cuenca López. Universidad de Huelva Jesús Estepa Giménez. Universidad de Huelva Rafael Feito Alonso. Universidad Complutense (Madrid)

Francisco José García Gallardo. Universidad de Huelva

Soledad García Gómez. Universidad de Sevilla J. Eduardo García Díaz. Universidad de Sevilla
Fernando Hernández Hernández. Universidad de Barcelona

Salvador Llinares Ciscar. Universidad de Alicante Alfonso Luque Lozano. Universidad de Sevilla Rosa Martín del Pozo. Universidad Complutense (Madrid)

José Martín Toscano. IES Fernando Herrera (Sevilla) Jaume Martínez Bonafé. Universidad de Valencia F. Javier Merchán Iglesias. Universidad de Sevilla Emilia Moreno Sánchez. Universidad de Huelva.Rosario Ortega Ruiz. Universidad de Córdoba

Antonio de Pro Bueno. Universidad de Murcia Fco. de Paula Rodríguez Miranda. Universidad de Huelva

Pedro Sáenz-López Buñuel. Universidad de Huelva Antoni Santisteban Fernández. Universidad Autónoma (Barcelona) Emilio Solís Ramírez. Catedrático de IES. $\mathbf{M}^{\mathbf{a}}$ Victoria Sánchez García. Universidad de Sevilla. Magdalena Suárez Ortega. Universidad de Sevilla

\section{Consejo asesor}

Manuel Área Moreira. Universidad de La Laguna

Jaume Carbonell. Director Cuadernos de Pedagogía. Barcelona

César Coll. Universidad de Barcelona

Christopher Day. Universidad de Nothingham. U.K.

Juan Delval. Universidad Nacional de Educación a Distancia

John Elliott. Universidad de East Anglia. Norwich. U.K.

José Gimeno Sacristán. Universidad de Valencia

André Giordan. Universidad de Paris VII y Ginebra

Francisco Imbernón. Universidad de Barcelona

Ángel Pérez Gómez. Universidad de Málaga

Rafael Porlán Ariza. Universidad de Sevilla

Francesco Tonucci. Instituto de Pedagogía del C.N.R. Roma

Jurjo Torres Santomé. Universidad de A Coruña 
